\title{
Tratamiento con análogos de la hormona liberadora de gonadotrofinas (aGnRH) en niñas, niños y adolescentes
}

\author{
Treatment with gonadotropin-releasing hormone analogs \\ (GnRHa) in childhood and adolescence
}

\section{Comité Nacional de Endocrinología}

a. Hospital Italiano de Buenos Aires, Ciudad Autónoma de Buenos Aires, Argentina.

b. Hospital de Niños Dr. Ricardo Gutiérrez, Ciudad Autónoma de Buenos Aires, Argentina.

c. Hospital General de Agudos Dr. Juan A. Fernández, Ciudad Autónoma de Buenos Aires, Argentina.

d. Hospital de Pediatría S.A.M.I.C Prof. Dr. Juan P. Garrahan, Ciudad Autónoma de Buenos Aires, Argentina.

e. Hospital Nacional Posadas, El Palomar, Argentina.

f. Clínica Universitaria Reina Fabiola, Universidad Católica de Córdoba, Córdoba, Argentina.

g. Hospital General de Agudos Dr. E. Tornú, Ciudad Autónoma de Buenos Aires, Argentina.

\section{Correspondencia:}

Elisabeth M. Boulgourdjian: eboulgourdjian@ hotmail.com

Financiamiento: Ninguno.

Conflicto de intereses: Ninguno que declarar.

Recibido: 10-8-2021 Aceptado: 18-8-2021

\section{RESUMEN}

Desde hace varias décadas, los análogos de la hormona liberadora de gonadotrofinas $(\mathrm{aGnRH})$ son el tratamiento de elección en la pubertad precoz central (PPC) en niñas y en niños. Causan una inhibición del eje hipotálamohipófiso-gonadal, disminuyen la secreción de gonadotrofinas, estradiol y testosterona; como consecuencia, producen una regresión de los caracteres sexuales secundarios durante el tratamiento. En los últimos años, estos análogos también se utilizan en adolescentes transgénero, en adolescentes y adultas jóvenes con enfermedades oncológicas, en algunas situaciones muy particulares en niños y niñas con talla baja, y en pacientes con trastornos del neurodesarrollo. En Argentina, los más utilizados son el acetato de triptorelina y el acetato de leuprolide en sus formas de depósito. Estos medicamentos han demostrado eficacia y seguridad. El objetivo de esta publicación es realizar una revisión y actualización del uso de los aGnRH en niños, niñas y adolescentes.

Palabras clave: hormona liberadora de gonadotrofinas, análogos y derivados, tratamiento, niño, niña, adolescente.

\section{ABSTRACT}

For several decades, gonadotropin releasing hormone analogs (GnRHa) are the medical treatment selected for central precocious puberty (CPP) in girls and boys. They generate an inhibition of the hypothalamus-pituitarygonadal axis decreasing LH, FSH, estradiol and testosterone secretion and, in this way, they produce a regression of secondary sexual characters under treatment. In the last years, these analogs are also used in trans adolescents, in adolescents and young adults with oncological diseases, in some very particular situations in children with short stature and in patients with neurodevelopmental disorders. In Argentina the most commonly used formulations are triptorelin and leuprolide acetate depot forms. These analogs have proven both their efficacy and their safety. The aim of this paper is to review and update about the use of GnRHa in children and adolescents.

Key words: Gonadotropin-releasing hormone, analogs and derivatives, treatment, child, adolescent.

http:/ / dx.doi.org/10.5546/ aap.2022.S1

Cómo citar: Comité Nacional de Endocrinología. Tratamiento con análogos de la hormona liberadora de gonadotrofinas (aGnRH) en niñas, niños y adolescentes. Arch Argent Pediatr 2022;120(1):S1-S8.

\section{INTRODUCCIÓN}

Los análogos de la hormona liberadora de gonadotrofinas (aGnRH) han sido ampliamente utilizados desde hace varias décadas en el tratamiento de la pubertad precoz central (PPC) en niñas y niños, y demostraron eficacia y seguridad. En los últimos años, el uso se ha extendido a otras situaciones clínicas en las que, por diferentes motivos, se requiere inhibir el eje hipotálamohipófiso-gonadal (HHG).

El objetivo de esta publicación es realizar una revisión y actualización sobre las diferentes indicaciones del uso de los aGnRH en pediatría y adolescencia.

Coordinación: Elisabeth M. Boulgourdjian

Guillermo Alonso $^{a}$ (D), Andrea Arcari ${ }^{b}$ (D),_Sonia V. Bengolea ${ }^{c}$ (D), Mariana Costanzo (D), Silvia D'Amato ${ }^{e}$, Ana Keselman ${ }^{b}$ (D), Silvia Martin $f$ (D), Viviana R. Pipman ${ }^{8}$ (D),María S. Rodríguez Azraks 


\section{GENERALIDADES}

La pubertad es un período de transición biológica de la niñez a la adultez en el que ocurre el desarrollo de los caracteres sexuales secundarios y que culmina con la adquisición de la capacidad reproductiva y el alcance de la talla adulta.

La pubertad se inicia con la reactivación del eje HHG. La secuencia de eventos que generan dicha activación está dada por modificaciones en neurotransmisores y neuropéptidos que inducen un incremento en la secreción pulsátil del factor liberador de gonadotrofinas $(\mathrm{GnRH})$ producido en el hipotálamo. El GnRH es un neuropéptido de 10 aminoácidos. Las neuronas secretoras que lo producen se originan en la placa olfatoria y migran hacia el hipotálamo durante el desarrollo fetal. Para lograr el efecto óptimo del GnRH sobre su receptor hipofisario, se requiere que el estímulo sea de manera pulsátil. De esta forma, los receptores se sintetizan aumentando su número y los que se encuentran enmascarados en la membrana se vuelven a hacer visibles mejorando así la respuesta; este mecanismo se conoce como up-regulation. Por el contrario, si la estimulación del GnRH es continua y homogénea, los receptores dejan de producirse y los presentes en la membrana se enmascaran, anulando así la respuesta. Este fenómeno es conocido como desensibilización o down-regulation. ${ }^{1}$

El incremento de la secreción pulsátil del GnRH induce, a su vez, la liberación pulsátil de gonadotrofinas hipofisarias: LH (hormona luteinizante) y FSH (hormona foliculoestimulante). En respuesta a las gonadotrofinas y a un complejo sistema de regulación autocrina y paracrina, las gónadas (testículos y ovarios) producen esteroides sexuales (testosterona y estradiol, respectivamente) los cuales ejercerán una retroalimentación negativa sobre el hipotálamo y la hipófisis (Figura 1).

El incremento de esteroides sexuales causa cambios físicos graduales que se acompañan de profundos cambios psicológicos y sociales. Estos últimos son el producto de la marcada modificación del esquema corporal que constituye el rasgo más visible de la pubertad. En las niñas, el primer signo de desarrollo puberal es la aparición del botón mamario (telarca) que, en nuestra población, se produce a una edad promedio de 10,8 años, con una variación individual entre 8,3 y 13,3 años. En los varones, el primer signo de inicio

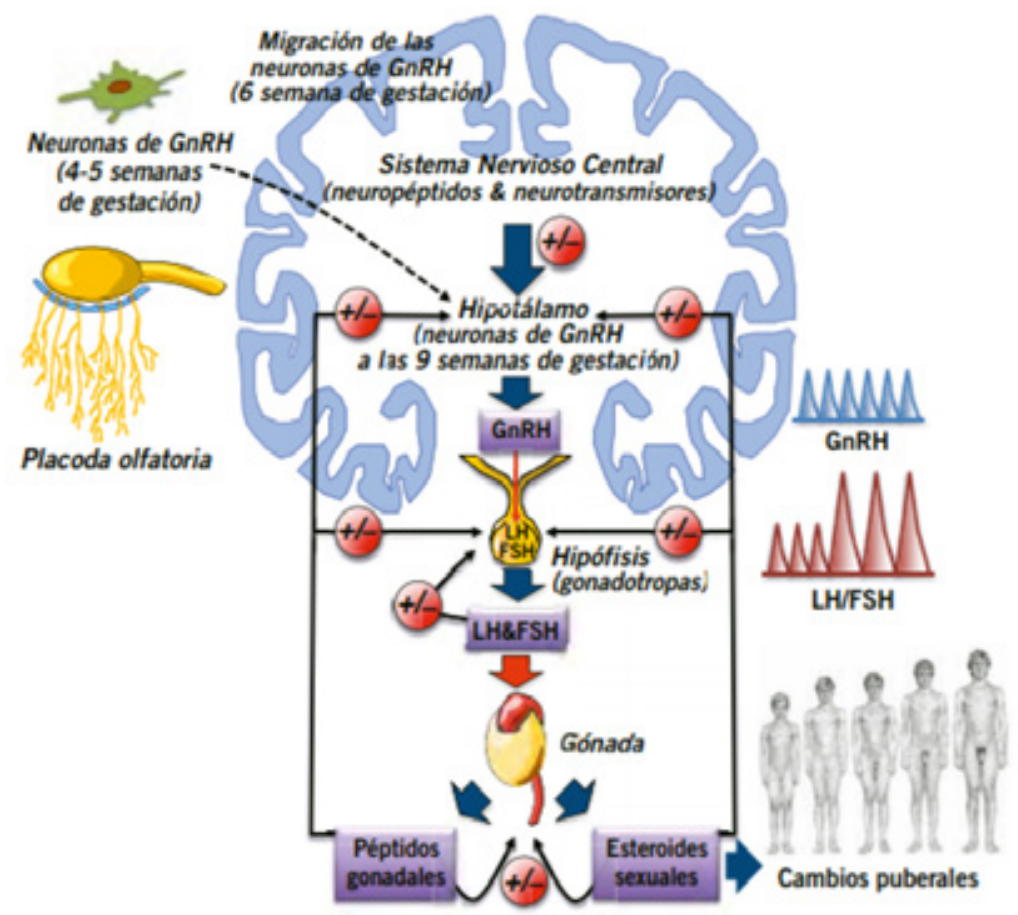


puberal es el aumento del volumen testicular, que alcanza o supera los $4 \mathrm{ml}$ a una edad promedio de 11,5 años, con límites entre 8,9 y 14,7 años. ${ }^{2}$ Los esteroides sexuales también causan un aumento significativo de la secreción hipofisaria de la hormona del crecimiento (HC), que ejerce una acción sinérgica en la promoción del crecimiento durante el empuje puberal.

Desde la década de los 80 , los análogos del GnRH se han constituido en el tratamiento de elección de la pubertad precoz central. Dichos compuestos se obtienen mediante modificaciones en la molécula del GnRH endógena que le confieren mayor potencia por su mayor afinidad de unión al receptor y mayor resistencia a la degradación enzimática. Estos fármacos producen una estimulación continua de las células gonadotropas hipofisarias, que conlleva una desensibilización de estas y, por ende, una disminución de la secreción de LH y, en menor medida, de FSH. Es así que actúan a nivel hipofisario por el mecanismo de desensibilización antes mencionado. Luego de un período inicial de liberación de gonadotrofinas, los niveles de LH y FSH disminuyen y, en consecuencia, también los esteroides sexuales; de esta manera han mostrado ser eficaces en inhibir el eje HHG.

A lo largo del tiempo, ha ido variando la forma de presentación de estos fármacos, así como la vía y el intervalo de administración. Es importante destacar que el efecto de dicho tratamiento es transitorio y reversible, es decir que, al suspenderlo, la pubertad progresa de forma normal. ${ }^{3}$

Los análogos más utilizados en nuestro país son el acetato de triptorelina y el acetato de leuprolide en sus formulaciones de depósito. Se administran por vía intramuscular cada 28 días (mensual) o cada 12 semanas (trimestral). Recientemente, ha ingresado al país una formulación de leuprolide de aplicación semestral que se administra por vía subcutánea (Tabla 1). Otra opción terapéutica es el implante de un
aGnRH llamado histerelina, que se administra por vía subcutánea en forma anual, el cual no se encuentra disponible en nuestro país. Los efectos adversos asociados a los análogos son muy poco frecuentes y no revisten gravedad (abscesos estériles en el sitio de aplicación, cefaleas y exantema cutáneo, entre otros). ${ }^{4}$

\section{TRATAMIENTO CON ANÁLOGOS DE GnRH EN NIÑAS Y NIÑOS CON PUBERTAD PRECOZ CENTRAL}

Se define como pubertad precoz (PP) la aparición de los caracteres sexuales secundarios antes de los 8 años en la niña y antes de los 9 años en el varón, acompañada de una aceleración de la velocidad de crecimiento y un avance de la maduración esquelética, que lleva al cierre prematuro de las epífisis, lo que suele resultar en una talla adulta menor a la talla media parental. ${ }^{5}$ Esta condición puede producir un impacto emocional importante, ya que la irrupción de la pubertad a temprana edad es un evento inesperado que, en ocasiones, genera angustia tanto a niñas y niños como a su entorno familiar. ${ }^{6}$ La PP es más frecuente en niñas que en niños en una relación 10 a 1 . En cuanto a los modos de presentación, puede ser esporádica o familiar.

La causa más frecuente de PP es la desencadenada por una activación prematura del eje HHG denominada pubertad precoz central (PPC). Otra causa menos frecuente de PP, independiente de la secreción de gonadotrofinas, es la denominada pubertad precoz periférica, causada por la secreción autónoma de esteroides sexuales por las gónadas o, menos frecuentemente, por las glándulas suprarrenales o por estrógenos exógenos.

Los aGnRH son el tratamiento de elección en la PPC, ya que, como se ha mencionado, su mecanismo de acción se produce a nivel hipofisario. Los aGnRH ocasionan una inhibición de los caracteres sexuales secundarios, disminuyen la velocidad de crecimiento y frenan

Tabla 1. Análogos de la hormona liberadora de gonadotrofinas

\begin{tabular}{lccc}
\hline Análogo del GnRH & Forma de presentación & Intervalo & Vía de administración \\
\hline Acetato de triptorelina & $3,75 \mathrm{mg}$ & Mensual & Intramuscular \\
& $11,25 \mathrm{mg}$ & Trimestral & Intramuscular \\
Acetato de leuprolide & $7,5 \mathrm{mg}$ & Mensual & Intramuscular \\
& $22,5 \mathrm{mg}$ & Trimestral & Intramuscular \\
& $45 \mathrm{mg}$ & Semestral & Subcutánea \\
\hline
\end{tabular}


el avance de la edad ósea, preservando de esta forma la talla adulta. Las menstruaciones se interrumpen en aquellas niñas que ya las hayan presentado. Asimismo, disminuye el impacto emocional que se produce en las niñas y en los niños que experimentan un desarrollo sexual precoz. Un pequeño número de niñas que inician el tratamiento con un desarrollo puberal muy avanzado pueden presentar, luego de la primera aplicación, un sangrado genital escaso y autolimitado debido al estímulo inicial que producen los aGnRH.

La duración del tratamiento debe ser individualizada, basándose en la edad cronológica, la evaluación de la talla, la evolución de la maduración esquelética y la adaptación psicosocial de las y los pacientes. En general, se acepta que el tratamiento sea suspendido alrededor de los 10-11 años en las niñas y entre los 12 y 13 años en los varones. Los signos puberales reaparecen de manera gradual y la menarca se presenta, en promedio, al año de la suspensión. ${ }^{7}$

En nuestro país, se llevó a cabo un trabajo en la División de Endocrinología del Hospital de Niños Dr. Ricardo Gutiérrez sobre 210 niñas con PPC, de las cuales 158 eran portadoras de una PPC idiopática. Todas fueron tratadas con acetato de triptorelina o acetato de leuprolide en forma de depósito a las dosis habituales. De esas 158 niñas, 76 alcanzaron la talla adulta, la cual fue de 160,4 $\pm 0,72 \mathrm{~cm}$ (puntaje Z: -0,01), muy similar a la talla media parental, que fue de 160,24 $\pm 0,62 \mathrm{~cm}$ (puntaje Z: -0,09). No se observaron efectos adversos de importancia. El tratamiento con aGnRH demostró ser eficaz y seguro para preservar la talla adulta. ${ }^{8}$

Algunos autores manifestaron preocupación por el potencial impacto de este tratamiento sobre el peso corporal. Sin embargo, la mayoría de los estudios a largo plazo mostraron una mayor prevalencia de sobrepeso y obesidad en pacientes con PPC en el momento del diagnóstico, pero sin cambios significativos en el índice de masa corporal (IMC) al final de tratamiento, independientemente del sexo, la edad de inicio de la pubertad y el inicio y la interrupción del tratamiento, y el tipo de aGnRH utilizado. ${ }^{7,9}$. Asimismo, se ha demostrado que los aGnRH no causan un impacto negativo sobre la densidad mineral ósea en las niñas con PPC. ${ }^{3}$

\section{TRATAMIENTO CON ANÁLOGOS DE GnRH EN ADOLESCENTES TRANSGÉNERO}

La identidad de género (IG) se define como la vivencia interna e individual del género tal como cada persona la siente, la cual puede corresponder o no con el sexo asignado al momento del nacimiento, en el que sobre la base de las características biológicas se asigna el sexo del recién nacido/a. La mayoría de las personas construyen su IG acorde a esta asignación y a su crianza. Aquellos niños, niñas y adolescentes que poseen una IG que no coincide con su sexo biológico pueden sentir disconformidad con el género asignado y reconocerse como personas transgénero, travestis o no binarias.

El reconocimiento de la IG comienza entre los tres y cinco años de edad, y se intensifica en la adolescencia. ${ }^{10}$ Existe poca información sobre la cantidad de niños y niñas en los que la disconformidad de género (DG) persiste en la vida adulta. La mayoría (75-80 \%) de los niños y niñas prepuberales que dicen sentirse no conformes con el sexo asignado al nacimiento no seguirán experimentando este sentimiento al llegar a la pubertad. ${ }^{10}$ Sin embargo, si la DG continúa luego del inicio de la pubertad, es muy probable que se mantenga en la edad adulta.

Los y las adolescentes que deseen realizar una transición hacia el género autopercibido podrán solicitar la inhibición de la pubertad, acompañando la firma del consentimiento informado (Ley 26743 de Identidad de Género, sancionada en Argentina en el año 2012, art. 11). La resolución 3159/19 establece una cobertura del $100 \%$ en insumos para hormonización, incluida la medicación utilizada para la inhibición del desarrollo puberal.

La intervención médica de forma precoz mediante la supresión del eje hipotálamohipófiso-gonadal (HHG) con el tratamiento con aGnRH, como triptorelina o leuprolide, permite prevenir los sentimientos de angustia o incomodidad que pudieran surgir asociados al desarrollo de los caracteres sexuales secundarios propios del sexo asignado al nacer. Esto favorece una adaptación psicosocial más adecuada, al atravesar un período de transición para vivir y ser aceptado/a como una persona del género deseado, y les permite a los y las adolescentes disponer de tiempo para la exploración de su identidad.

Como ya hemos mencionado, estos fármacos han constituido el tratamiento de elección en la pubertad precoz desde hace varias décadas; 
múltiple evidencia demuestra su eficacia y su seguridad. No obstante, se requieren estudios de seguimiento que evalúen la utilización en el marco de los procesos de construcción corporal.

El uso de los aGnRH produce una regresión o detención de los cambios puberales en función del nivel de desarrollo que presenten al momento de inicio del tratamiento. ${ }^{12}$ Por tal motivo, se recomienda comenzar la supresión en etapas tempranas del desarrollo puberal, durante el estadio de Tanner II, a fin de evitar la aparición de cambios corporales irreversibles.

Si el/ la adolescente quisiera reanudar la progresión de su desarrollo puberal por acción de las hormonas gonadales endógenas, se deberá interrumpir el uso de los aGnRH en el momento en que lo solicite. Dado que la supresión que provocan es reversible, los cambios puberales progresarán a los pocos meses de la suspensión del tratamiento. ${ }^{12}$

Durante el tratamiento con aGnRH en adolescentes transgénero mujeres, se logra el cese de las erecciones, se detiene la virilización y disminuye el volumen testicular. Si el tratamiento se inicia con la pubertad avanzada, no se logra la reversión de algunas características, como la prominencia de la nuez de Adán, la voz grave, la configuración ósea masculina (mandíbula grande, pies y manos grandes, estatura alta) y el patrón del vello masculino en la cara y en las extremidades. En cuanto al tiempo de tratamiento con aGnRH, algunos autores proponen mantenerlo 6 meses y luego asociar un antiandrógeno (espironolactona o acetato de ciproterona) al tratamiento de hormonización cruzada con estrógenos. ${ }^{12,13}$ Otros sugieren que el análogo sea mantenido de por vida o hasta la gonadectomia en las mujeres transgénero, debido a que las dosis de estrógenos necesarias para suprimir los niveles de testosterona son muy altas, lo que implica un alto riesgo de efectos secundarios, como por ejemplo tromboembolismo. ${ }^{14}$

En adolescentes transgénero varones, el tejido mamario se vuelve atrófico y se inhiben los ciclos menstruales, llevando a la amenorrea. Se suele continuar con el bloqueo del eje HHG durante los primeros 6 a 12 meses de iniciado el tratamiento de hormonización cruzada con testosterona, momento en el cual las concentraciones de testosterona son lo suficientemente altas como para inhibir la secreción endógena de estrógenos.

Se logra mejor inhibición con el uso de aGnRH de administración mensual por vía intramuscular. En caso de que no se logre suprimir por completo el eje gonadal, es posible acortar el intervalo de administración para lograr mayor efectividad.

Los aGnRH se consideran medicamentos seguros, ya que, como se ha mencionado, existen pocos efectos adversos relacionados con ellos. Se ha observado aumento de la masa grasa y disminución de la masa magra, hipertensión arterial, aparición de sofocos, fatiga y alteraciones del humor como consecuencia de la supresión de la pubertad si el tratamiento es tardío, pero son excepcionales si el bloqueo es precoz. Dado que la pubertad es un momento crítico para la adquisición de la masa ósea, ha sido descripto también un efecto negativo sobre la mineralización ósea, con mejoría tras iniciar la terapia con hormonas sexuales. No han sido descriptas modificaciones en el perfil lipoproteico, insulina, glucosa, función hepática y renal.

\section{TRATAMIENTO CON ANÁLOGOS DE GnRH EN NIÑAS, NIÑOS Y ADOLESCENTES CON TALLA BAJA}

La ganancia de talla desde el inicio del empuje puberal hasta la finalización del crecimiento es de $24-30 \mathrm{~cm}$ en los varones y de $23-27 \mathrm{~cm}$ en las mujeres, lo cual constituye solo el 15-20\% de la talla adulta, mientas que el $75-80 \%$ restante va a estar determinado por el crecimiento prepuberal. Por ello, existe una fuerte correlación positiva entre la talla al inicio de la pubertad y la talla adulta. De este modo, surge la importancia de que, en el momento en que los niños y niñas inician la pubertad, hayan alcanzado una talla suficiente como para que les permita adquirir una talla adulta acorde a su talla media parental.

Es difícil establecer cuál es la talla óptima de inicio de pubertad, la cual también depende de la población referida. En la población japonesa, la talla al inicio de la pubertad en niños sanos es de aproximadamente $145 \mathrm{~cm}$ en varones ${ }^{15}$ y $135 \mathrm{~cm}$ en mujeres. ${ }^{16}$ Tanaka y col., han demostrado que los niños que inician la pubertad con una talla inferior a $135 \mathrm{~cm}$ (varones) y 132,5 cm (mujeres) tendrían dificultades para llegar a una talla adulta mayor a $160 \mathrm{~cm}$ y $150 \mathrm{~cm}$ respectivamente. ${ }^{17}$ Es por ello que las niñas y los niños que inician la pubertad a una edad normal o temprana, pero con una talla baja constituyen un grupo de riesgo de alcanzar una talla adulta baja.

Por tal motivo, se ha propuesto la utilización de moduladores de la pubertad en niños y niñas con talla baja de diferentes causas (deficiencia de hormona de crecimiento, talla baja idiopática, pequeños para edad gestacional [PEG]) entre 
otras, con el objetivo de retrasarla y así dar mayor tiempo al crecimiento prepuberal. Sin embargo, el beneficio de la utilización de estos moduladores sobre la talla adulta no ha sido claramente demostrado.

Los estudios disponibles hasta el momento indican que los aGnRH administrados aisladamente en pacientes con talla baja y pubertad normal o adelantada, pese a inhibir el desarrollo puberal y prolongar el período de crecimiento, solo son útiles en algunos casos individuales para mejorar la talla adulta. ${ }^{3}$ Es por ello que algunos autores han propuesto la asociación de hormona de crecimiento y análogos de $\mathrm{GnRH}$, que parece mostrar mayores beneficios. Hasta el momento, los resultados de dichos tratamientos no son del todo concluyentes, ya que se observa una variación considerable en la respuesta a estos. ${ }^{18}$

Los niños y niñas nacidos PEG merecen una mención especial. La mayoría de ellos recuperan el déficit de peso y talla entre los 2 y 3 años de edad, mientras que alrededor del 10-15\% no presenta crecimiento compensatorio (catch-up) y permanecen con una talla baja durante la infancia y la vida adulta. La edad de inicio puberal es relativamente temprana; el pico de velocidad de crecimiento también es más temprano y de menor amplitud, y la maduración esquelética es acelerada, lo que lleva a una fusión epifisaria precoz. Todo lo anteriormente mencionado asociado a la talla baja de inicio puberal hace que la talla adulta se vea adversamente afectada en relación con la talla media parental.

Si bien los datos disponibles hasta el momento son limitados, algunos autores sugieren que el tratamiento combinado, hormona de crecimiento y aGnRH, podría incrementar la talla adulta en los niños y niñas que inician la pubertad con una talla baja. ${ }^{19}$

\section{TRATAMIENTO CON ANÁLOGOS DE GnRH EN ADOLESCENTES CON PATOLOGÍA ONCOLÓGICA}

El desarrollo de nuevos tratamientos ha cambiado favorablemente el pronóstico de muchas enfermedades neoplásicas; en consecuencia, el número de sobrevivientes de cáncer ha ido en aumento. Más del $80 \%$ de todos los pacientes diagnosticados con cáncer antes de los 15 años tiene en la actualidad una sobrevida de más de 5 años y se espera que la mayoría de ellos sobrevivan muchos años más después del tratamiento..$^{20}$ En Argentina, anualmente se diagnostican 1400 nuevos casos de cáncer en niños menores de 15 años: las leucemias, linfomas y tumores del sistema nervioso central son los más frecuentes. ${ }^{21}$

Los resultados positivos derivados de una terapéutica más efectiva con el uso de distintos agentes quimioterápicos ha puesto en evidencia los efectos adversos a corto y largo plazo. Mientras que el daño citotóxico es mayormente reversible en tejidos de rápida división celular, como la médula ósea, el tracto gastrointestinal y el timo, estos cambios parecen ser progresivos e irreversibles en otros sistemas. Así, en el ovario, donde el número de células germinales es limitado, estos abordajes terapéuticos desencadenan trastornos que se manifiestan de diferentes formas clínicas, desde cambios menores, como alteraciones del ciclo menstrual, hasta la infertilidad. ${ }^{22}$ Dentro del folículo ovárico, tanto los ovocitos como las células de la granulosa son vulnerables al daño causado por la quimioterapia (QMT). ${ }^{23}$

Con el objetivo de preservar la fertilidad futura en pacientes que reciben tratamiento antineoplásico, se han implementado distintas estrategias. Debido a que las células en división mitótica son más sensibles a los efectos deletéreos de los agentes quimioterápicos, se ha sugerido que la inhibición del eje HHG con aGnRH produce un estado hipogonadotrófico que disminuye el número de folículos primordiales que entran en maduración a estadios más vulnerables a la QMT. Además, el hipoestrogenismo provoca disminución de la perfusión útero-ovárica y, por ende, disminución del ingreso de los agentes QMT al ovario; por lo tanto, el epitelio germinal se haría menos susceptible a los efectos de la QMT. ${ }^{24}$

Desde hace más de 30 años se ha utilizado el tratamiento con aGnRH previo al inicio y durante la QMT para minimizar el riesgo de insuficiencia ovárica. Varias revisiones sistemáticas y metanálisis muestran una mayor tasa de recuperación de ciclos y función ovárica después de la QMT en pacientes tratadas con aGnRH antes y durante la quimioterapia que en aquellos grupos sin tratamiento. ${ }^{25-27}$ Sin embargo, aún es controvertida la utilidad del tratamiento con aGnRH en la preservación de la fertilidad durante la QMT. ${ }^{28-30,3}$

La metrorragia secundaria a plaquetopenia es una complicación importante en las adolescentes y adultas jóvenes que son sometidas a tratamientos mielosupresivos. Meirow y col., demostraron la 
utilidad de los aGnRH en reducir la metrorragia moderada o grave en todas las pacientes tratadas; la incidencia en el grupo no tratado fue del $40 \%{ }^{28}$

\section{TRATAMIENTOS CON ANÁLOGOS DE GnRH EN NIÑAS, NIÑOS Y ADOLESCENTES CON DISCAPACIDAD}

La etapa de la pubertad en pacientes con discapacidad puede ser particularmente estresante, especialmente para las familias. La preocupación está centrada en algunas situaciones que pueden presentarse como cambios de humor, agresividad y comportamiento sexual inapropiado, entre otras. En las niñas, también se plantean interrogantes acerca del manejo de las menstruaciones y la higiene menstrual, síntomas asociados a la menstruación, como dolor o incomodidad, y riesgo de abuso sexual. ${ }^{31}$ Es por ello que se ha propuesto el uso de aGnRH en algunos pacientes, aunque hasta el momento no hay evidencias suficientes que avalen su utilización. ${ }^{3,32}$

\section{CONCLUSIONES}

Dada la amplia experiencia en la utilización de aGnRH, se considera un tratamiento eficaz y seguro, que permite la inhibición transitoria y reversible del desarrollo puberal. En niñas, niños $\mathrm{y}$ adolescentes, sus principales indicaciones son la inhibición puberal en PPC, en adolescentes transgénero, así como la inhibición puberal transitoria en adolescentes mujeres con patología oncológica. Pueden ser considerados de utilidad en niños y niñas que presentan una pubertad rápidamente progresiva con talla baja de inicio asociada a mal pronóstico de estatura adulta, $y$ en algunas ocasiones en niñas y niños con discapacidad.

Se destaca el rol de la pediatría, con su derivación oportuna ante los primeros cambios puberales, y también el trabajo conjunto con endocrinología para favorecer la adherencia y el cumplimiento del tratamiento de los niños, niñas y adolescentes, optimizando así su efectividad. En todos los casos, el tratamiento debe ser individualizado y adecuarse a las necesidades de los y las pacientes, y de sus familias.

\section{REFERENCIAS}

1. Scacchi P. Fisiopatología Molecular y Clínica Endocrinológica. Sección V. Neuroendocrinología: Hipófisis e Hipotálamo. En: Calandra RS, Barontini MB (eds). Fisiopatología Molecular y Clínica Endocrinológica. Buenos Aires: Eli Lilly; 2015.Págs.229-42.

2. Lejarraga H, Cusminsky M, Castro EP. Age of onset of puberty in urban argentinian children. Ann Hum Biol. 1976;
3(4):379-81.

3. Bangalore Krishna K, Fuqua JS, Rogol AD, Klein KO, et al. Use of Gonadotropin-Releasing Hormone Analogs in Children: Update by an International Consortium. Horm Res Paediatr. 2019; 91(6):357-72.

4. Soriano-Guillén L, Argente J. Central precocious puberty, functional and tumor-related. Best Pract Res Clin Endocrinol Metab. 2019; 33(3):101262.

5. Larsen PR, Kronenberg HM, Melmed S, Polansky KS. Puberty: ontogenety, neuroendocrinology, physiology and disorder. In: Larsen PR, Kronenberg HM, Melmed S, Polonsky KS (eds). Williams textbook of endocrinology. 10th ed. Philadelphia: Saunders; 2003.Págs.1115-239.

6. Kim EY,LeeMI. Psychosocial aspects in girls with idiopathic precocious puberty. Psychiatry Investig. 2012; 9(1):25-8.

7. Guaraldi F, Beccuti G, Gori D, Ghizzoni L. Management of endocrine disease: Long-term outcomes of the treatment of central precocious puberty. Eur J Endocrinol. 2016; 174(3):R79-87.

8. Escobar de Lázzari ME, Gryngarten MG, Ropelato MG, Arcari AJ, et al. Pubertad Precoz en Niñas. Separata Montpellier 2013. [Acceso: 20 de agosto de 2021]. Disponible en: https: / / www.montpellier.com.ar/Uploads / Separatas /Separata $\% 2036 \% 20$ Pubertad $\% 20$ Precoz $\% 20$ en $\% 20$ ni\%C3\%B1as\%20B.pdf

9. Arcari AJ, Gryngarten MG, Freire AV, Ballerini MG, et al. Body mass index in girls with idiopathic central precocious puberty during and after treatment with GnRH analogues. Int J Pediatr Endocrinol. 2016; 2016:15.

10. Olson J, Schrager SM, Belzer M, Simons LK, Clark L. Baseline Physiologic and Psychosocial Characteristics of Transgender Youth Seeking Care for Gender Dysphoria. J Adolesc Health. 2015; 57(4):374-80.

11. Fernández Mentaberry V, Pipman V, Suárez M, Boquete $\mathrm{C}$, et al. Herramientas de abordaje ante la consulta de diversidad sexual en la infancia y adolescencia. Rev Argent Endocrinol Metab. 2020; 57(4):31-40.

12. Argentina. Ministerio de Salud. Atención de la salud integral de personas trans, travestis y no binarias. Guía para equipos de salud. 2020. [Acceso: 20 de agosto de 2021]. Disponibleen:https:/ / bancos.salud.gob.ar/sites/default/ files / 2020-10/ guia-salud-personas-trans-travestis-nobinarias.pdf

13. Telfer MM, Tollit MA, Pace CC, Pang KC. Australian Standards of Care and Treatment Guidelines for Trans and Gender Diverse Children and Adolescents Version 1.1. Melbourne: The Royal Children's Hospital; 2018.

14. Hembree WC, Cohen-Kettenis PT, Gooren L, HannemaSE, et al. Endocrine Treatment of Gender-Dysphoric/GenderIncongruent Persons: AnEndocrineSociety Clinical Practice Guideline. J Clin Endocrinol Metab. 2017; 102(11):3869-903.

15. Fujieda K, Matsuura N. Growth and maturation in the male genitalia from birth to adolescence. I. Change of testicular volume. Acta Paediatr Jpn. 1987; 29(2):214-9.

16. Tanaka T, Imai T. The standard of breast development in normal girls: Diagnostic criteria for precocious puberty. J Child Health. 2005; 64:33-8 (in Japanese).

17. Tanaka T, Naiki Y, Horikawa R. Clinical factors related to the short adult height: early puberty for height. J Jpn Assoc Hum Auxol. 2011; 17:17-23.

18. Pozo Roman J. Crecimiento normal y baja talla. Pediatr Integral. 2015; 19(6):411.e1-23.

19. Lem AJ, van der Kaay CM, de Ridder MAJ, Bakker-van Waarde WM, et al. Adult Height in Short Children Born SGA Treated with Growth Hormone and Gonadotropin Releasing Hormone Analog: Results of Ramdomized, Dose-Response GH Trial. J Clin Endocrinol Metab. 2012; 97(11):4096-105. 
20. Phillips S, Padgett L, Leisenring W, Stratton KK, et al. Survivors of childhood cancer in the united states: prevalence and burden of morbidity. Cancer Epidemiol Biomarkers Prev. 2015; 24(4):653-63.

21. Moreno F, Chaplin MA. Registro oncopediátrico hospitalario argentino. 6a ed. Ciudad Autónoma de Buenos Aires: Instituto Nacional del Cáncer; 2018.

22. Chow JE, Stratton KL, Leisenring WM, Oeffinger K, et al. Pregnancy after chemotherapy in male and female survivors of childhood cancer treated between 1970 and 1999: a report from the Childhood Cancer Survivor Study cohort. Lancet Oncol. 2016; 17(5):567-76.

23. Bedoschi G, NavarroPA, Oktay K. Chemotherapy-induced damage to ovary: mechanisms and clinical impact. Future Oncol. 2016; 12(20):2333-44.

24. Dada T, Salha O, Allgar V, Sharma V. Utero-ovarian blood flow characteristics of pituitary desensitization. Hum Reprod. 2001; 16(8):1663-70.

25. Blumenfeld Z. Preservation of ovarian function and minimizing premature ovarian failure during chemotherapy using gonadotropin-releasing hormone analogs. Womens Health (Lond.) 2011; 7(6):635-40.

26. Hickman LC, Llarena NC, Valentine LN, Liu X, Falcone T. Preservation of gonadal function in women undergoing chemotherapy: a systematic review and meta-analysis of the potential role for gonadotropin-releasing hormone agonists. J Assist Reprod Genet. 2018; 35(4):571-81.

27. Meli MC, Caruso-Nicoletti M, La Spina M, Lo Nigro L, et al. Triptorelin for Fertility Preservation in Adolescents Treated With Chemotherapy for Cancer. J Pediatr Hematol Oncol. 2018; 40(4):269-76.

28. Meirow D, Rabinovici J, Katz D, Reuven O, et al. Prevention of Severe Menorrhagia in Oncology Patients with Treatment-Induced Thrombocytopenia by Luteinizing Hormone-Releasing Hormone Agonist and Depo-Medroxyprogesterone Acetate. Cancer. 2006; 107(7):1634-41.

29. Oktay K, Harvey BE, Partridge AH, Quinn GP, et al. Fertility Preservation in Patients With Cancer: ASCO Clinical Practice Guideline Update. J Clin Oncol. 2018; 36(19):19942001.

30. Sugishita $Y$, Suzuki N. Fertility preservation for adolescent and young adult cancer patients in Japan. Obstet Gynecol Sci. 2018; 61(4):443-52.

31. Zacharin M, Savasi I, Grover S. The impact of menstruation in adolescents with disabilities related to cerebral palsy. Arch Dis Child. 2010; 95(7):526-30.

32. Coshway L, Broussard J, Acharya K, Fried K, et al. Medical Therapy for Inappropriate Sexual Behaviors in a Teen with Autism Spectrum Disorder. Pediatrics. 2016; 137(4):e20154366. 